\title{
Rapid maxillary expansion treatment could produce long-term dental arch changes
}

\author{
Are there any long-term dental arch changes after rapid maxillary expansion \\ treatment in patients who have constricted arches?
}

\begin{abstract}
Lagravere MO, Major PW, Flores-Mir C. Long-term dental arch changes after rapid maxillary expansion treatment: a systematic review. Angle Orthod 2005; 75: 151-157
\end{abstract}

Data sources Medline, Medline In-Process, LILACS (Latin American and Caribbean Health Sciences Literature), PUBMED, Embase, Web of Science and the Cochrane Library were searched. Search terms were rapid palatal expansion or rapid maxillary expansion (RME) and tooth or dental changes. Reference lists from retrieved articles were also examined. Study selection For inclusion, an article had to satisfy the following criteria: describe a controlled clinical trial; have dental arch measurements made for cephalometric radiographs or dental casts; and include no surgical treatment that could affect RME effects during the evaluation period. Studies without an adequate control to factor out normal growth changes were excluded.

Data extraction and synthesis Selected articles were independently evaluated by three researchers. Interexaminer discrepancies were resolved by discussion to reach a consensus. A summary of sample size, retention period, measurement error and evaluation method were listed in a table.

Results The search identified 164 articles, of which four met the inclusion criteria. From the final four articles, two measured changes using dental casts and two assessed changes using radiographs (one with lateral cephalometric radiographs and one with posteroanterior radiographs). Based on direct model treatment, clinically significant long-term maxillary molar width increase $(3.7-4.8 \mathrm{~mm})$ could be achieved. Because of crown tipping, the reported long-term increase varied according to the reference point used for measurements. The range of maxillary cuspid arch width expansion was more consistent and was similar for adolescents and adults $(2.2-2.5 \mathrm{~mm})$. Less mandibular molar and cuspid arch width expansion was attained in adults compared with adolescents. A significant overall gain was found in the maxillary $(6 \mathrm{~mm})$ and mandibular $(4.5 \mathrm{~mm})$ arch perimeter in adolescents treated with RME and edgewise appliances. When comparing radiographically the early-treated and late-treated groups with their respective controls, there was a significant gain of the maxillary intermolar width ( 2.7 and $3.5 \mathrm{~mm}$, respectively) in both treated groups. No statistically or clinically significant differences were found in molar vertical position or in incisor inclination.

Conclusions Similar maxillary molar and cuspid expansion could be found in adolescents and young adults. Significantly less indirect mandibular molar and cuspid expansion was attained in young adults compared with adolescents. A significant overall gain in the maxillary and mandibular arch perimeter was found in adolescents. More transverse dental arch changes were found after puberty than before, but the difference may not be clinically significant. No anteroposterior dental changes were found on lateral cephalometric radiographs.

Address for correspondence: Carlos Flores-Mir, Faculty of Medicine and Dentistry, Room 4051A, Dentistry/ Pharmacy Centre, University of Alberta, Edmonton, Alberta, Canada T6G 2N8. E-mail: carlosflores@ualberta.ca

\section{Commentary}

RME is recognised as a cornerstone of the early orthopaedic expansion protocol for treating people who have arch length discrepancy. It has been widely used and studied for more than 40 years. Until now, no conclusive long-term results have been reported on either dental or skeletal changes after RME treatment.

This article was intended to be a systematic review of the dental changes. To differentiate their review from the previous studies, the authors repeatedly mentioned the previous two meta-analyses and one systematic review, stating that the main disadvantage of these was the absence of a control group to factor out the normal growth changes. During normal growth, it is known that both maxillary and mandibular transpalatal widths increase, from the early mixed dentition to the permanent dentition, ${ }^{1}$ and arch length decreases in young adults possibly due to the anterior component of occlusal force. ${ }^{2,3}$ These changes may not be significant during the expansion period, but may be so during the retention period. Considering, however, that the retention period in the included studies varies from 3-8 years, and that the controls in each study are different (the studies by McNamara ${ }^{4}$ and Baccetti ${ }^{5}$ et al used the Michigan growth study group as controls: the other two studies used a control group paired according to age), the results of this study might not be expected to be very different from the previous ones. This is especially so when only four studies were included, each of which is different from the rest in the expansion degree (would it be wiser to measure proportion change instead of $\mathrm{mm}$ change?); ${ }^{6}$ retention regime (it is known that the amount of relapse is related to the retention procedure after expansion); ${ }^{7}$ evaluation methods (results from different evaluation methods cannot be directly compared or combined); and controls (controls are important, but when each study defines its own control, the net results could hardly be compared between studies).

The only thing these four studies have in common is that they all use a Haas-type expander followed by fixed edgewise appliance. However, this raises another question how to discriminate between the dental changes by RME or by the fixed appliance. Compared with the previous studies, the quality of the literature search (which includes non-English literature) and the attention given to controls are the strong points in this study and are worthy of appreciation. Nevertheless, the results remain inconclusive and do not add new knowledge on the long-term effect of RME because they are based on very limited studies of second-level evidence.

\footnotetext{
Yijin Ren

Orthodontic Department, University of Gröningen, Gröningen, The Netherlands

1. Moyers RE, van der Linden FPGM, Riolo ML, et al. Standards of Human Occlusal Development. Monograph 5. Craniofacial Growth Series, Ann Arbor: Center for Human Growth and Development, University of Michigan; 1976.

2. Carter GA, McNamara JA. Longitudinal dental arch changes in adults. Am J Orthod Dentofacial Orthop 1998; 114:88-99.

3. Tibana RH, Palagi LM, Miguel JA. Changes in dental arch measurements of young adults with normal occlusion — a longitudinal study. Angle Orthod 2004; 74:618-623.
} 
4. McNamara Jr JA, Baccetti T, Franchi L, Herberger TA. Rapid maxillary expansion followed by fixed appliances: a long-term evaluation of changes in arch dimensions. Angle Orthod 2003; 73:344-353.

5. Baccetti T, Franchi L, Cameron CG, McNamara Jr JA. Treatment timing for rapid maxillary expansion. Angle Orthod 2001; 71:343-350.

6. Lima AL, Lima Filho RM, Bolognese AM. Long-term clinical outcome of rapid maxillary expansion as the only treatment performed in Class I malocclusion. Angle Orthod 2005; 75:416-420.
7. Hicks EP. Slow maxillary expansion. A clinical study of the skeletal versus dental response to low-magnitude force. Am J Orthod 1978; 73:121-141.

Evidence-Based Dentistry (2005) 6, 93-94.

doi:10.1038/sj.ebd.6400358

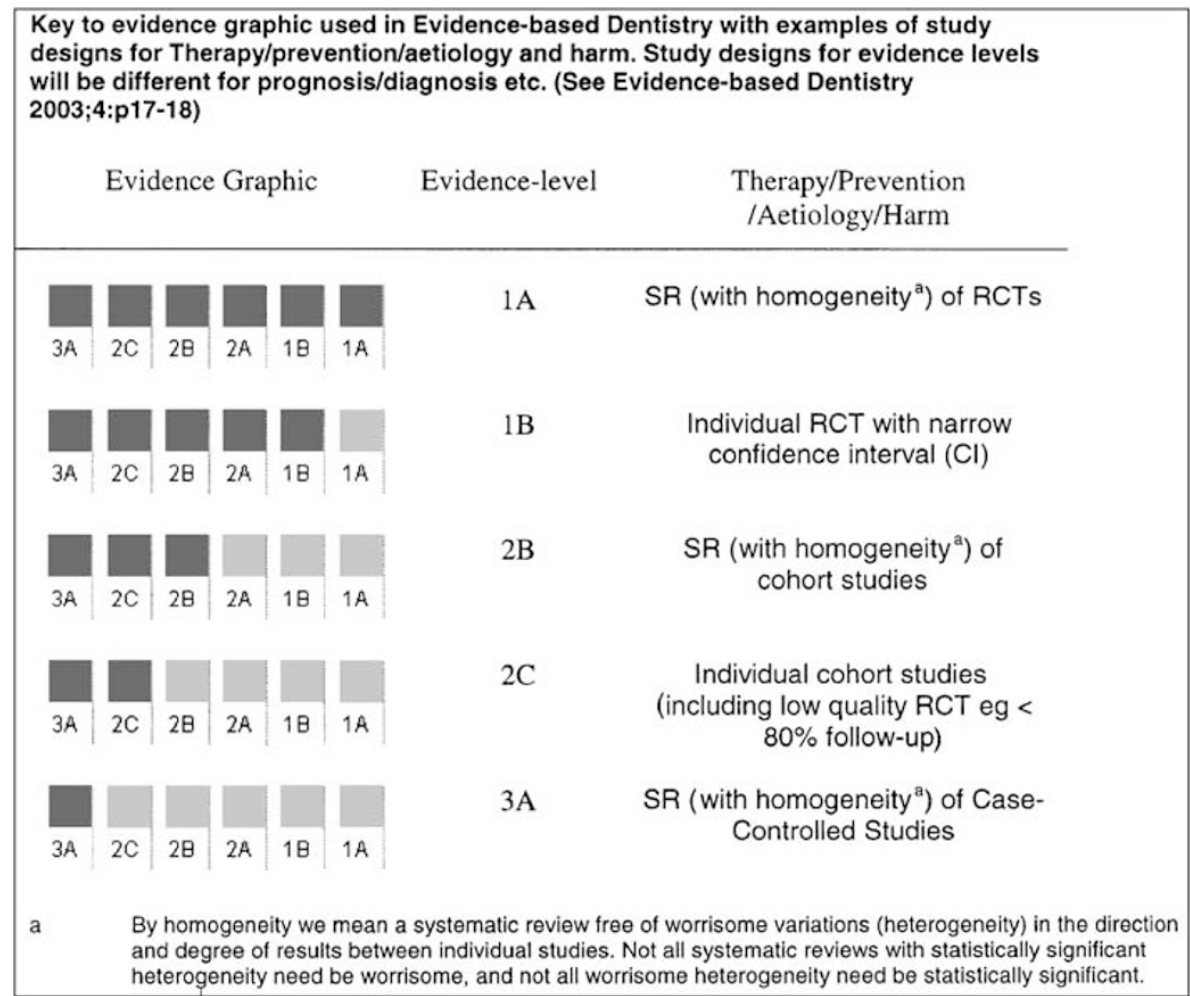

